\title{
MS13-P04 | Small-Angle X-RAy SCATtering Studies on the Interaction of Bicelles With Bovine Serum Albumin
}

Lin, Tsang-Lang (National Tsing Hua University, Hsinchu, TWN)

It is essential to understand how bicelles interact with proteins in order to develop bicelle drug delivery systems. In this study, the interaction of cationic lipid bicelles with the Bovine Serum Albumin (BSA) was investigated by smallangle X-ray scattering (SAXS). Disc-shaped bicelles can be formed spontaneously by mixing long-chain lipids with short-chain lipids at suitable ratios [1]. The long-chain lipids form the bilayer core of the bicelle while the shortchain lipids form the protecting rim of the bicelle. The surface charge of such bicelles can be varied by doping with cationic lipids, such as DC-Cholesterol, to form cationic bicelles [2]. For the cationic bicelles consist of $8.5 \mathrm{mM}$ DPPC, $3.3 \mathrm{mM}$ diC7PC and $1.5 \mathrm{mM} \mathrm{DC}$-cholesterol, at low BSA concentrations of $0.029 \mathrm{mM}$ to $0.058 \mathrm{mM}$, the presence of BSA induces the bicelles to form long stacking lamellar structure by encapsulating the BSA molecules between the lipid bilayers. When the BSA concentration is further increased above $0.072 \mathrm{mM}$, the lamellar structure is transformed to a rectangular column structure. It is likely that the mixed bilayers become modulated due to the strong charge interaction between the cationic lipid biclayers and the BSA molecules at high BSA concentrations.

[1] Yang, P.-W.; Lin, T.-L.; Hu, Y. \& Jeng, U-S. (2012) Chinese J. of Phys., 50, 349.

[2] Yang, P.-W.; Lin, T.-L.; Hu, Y. \& Jeng, U-S. (2013) Soft Matter, 9, 11542. 